\title{
A COMPARATIVE STUDY OF THE SAUDI ARABIA FLORA, MEDICINAL PLANTS OF AL- MANDAQ PROVINCE, AL-BAHA REGION.
}

\author{
Mohamed Elsayed Abdelhady*1,3 and Ahmed Gadim Mohamed ALI ${ }^{2,4 .}$ \\ ${ }^{1}$ Department of Biology, Faculty of Sciences \& Arts, Al Mandaq, Al-Baha University, \\ ${ }^{2}$ Department of Chemistry, Faculty of Sciences \& Arts, Al Mandaq, Al-Baha \\ University, \\ ${ }^{3}$ Department of Botany, Faculty of Sciences, Cairo, Al Azhar University, Egypt, \\ ${ }^{4}$ Department of Chemistry, Faculty of Education, Peace University, Sudan. \\ *Corresponding author: E. mail: elhadymohamed566@yahoo.com
}

\begin{abstract}
Medicinal and aromatic plants are important sources for the production of many pharmaceutical compounds such as antibiotic drugs. The presented study aims to survey and record the various medicinal plant species in Al-Mandaq Governorate - Al-Baha region - Kingdom of Saudi Arabia with a number of targeted locations such as Mashnaih, Darake and Khleb valleys, Medhas and Darake Dams in 2018 and 2019. 160 species of vascular plants belonging to 52 plant families were surveyed and recorded. The study proved that the Poaceae family is the most contain of the plant species (22 species, 13.7\%), followed by the Asteraceae family (19 species, 11.8\%), then the Fabaceae family (11 species, 6.8\%). According to the life forms, the annual plants represent the highest percentage among the recorded plants (56 species, 35\%), followed by the perennial herbs (48 species, $30 \%)$, then shrubs (26 species, 16.25\%) and the least recorded species are bi-perennials (5 species, $3.12 \%$ ), Palms and cactus (one species, $0.6 \%$ ). The recorded results and statistics represent a database of the floral content in the study area and also provide important information for the recorded plants and their uses in the fields of food, ornamental and biological applications.
\end{abstract}

Key words: Medicinal plants, Flora, Urban Flora, Saudi Arabia, Economic Uses, AlBaha Region. 


\section{Introduction}

By the middle of the nineteenth Century, at least $80 \%$ of all medicines derived from different plants. Then, after the scientific revolution, which leads to development of the pharmaceutical industry, the synthetic drugs dominated (Gilani and Atta-urRhaman, 2005). The using of plant species is the oldest medical science historically, it was heavily used in ancient times and the middle but even in our modern age, according to the reports, there are more than $70 \%$ of the Earth's population trust in the use of medicinal plants in the treatment and healing of various diseases that infect them with keeping in mind that there are some medicinal plants has harmful side effects, (Yuan, 2011). There are over 2.75,000 species of flowering plants known in the world today (Anonymous, 2000). Various plants and their parts used by man for the treatment of several diseases, particularly those caused by microorganisms. There is likelihood that all these plants used by the tribal people must have antimicrobial activities. A large number of antimicrobial agents already existing for various purposes have proved ineffective on target microorganisms (Babalola, 1988).

Medicinal plants play a significant role in providing primary health care services to the people (Rowinsky, et al., 1992). They serve as important therapeutic agents as well as important raw materials for the manufacture of traditional and modern medicines. It estimated that more than $25 \%$ of all prescription drugs used in the industrialized countries contain active principles that still extracted from plants including anticancer drugs (Shoeb, 2006).

Saudi Arabia covers an area of about 2,250,000 sq. km. the vast landscape is composed of a variety of habitats such as mountains, valleys, sandy and rocky deserts, meadows, and salt marshes. It has a hot desert climate and rainfall is scarce in most parts of the country. The flora of Saudi Arabia as well as the other countries in the Arabian Peninsula been neglected for a long time due to its arid climate. The first attempt to cover the flora of Saudi Arabia was in 1974 (Alfarhan et al., 1998). Many areas in Saudi Arabia, including Al-Baha region are rich in medicinal plants that traditionally used in treatment of some human and animal disease. This activity against diseases mainly attributed to presence of bioactive compounds in these plants; such as saponins, tannins, terpenoids, alkaloids, flavonoids, phenols and quinones. The percentage of endemic plants in Saudi Arabia is very low. About 50 species (2\%) are considered endemic to this country compared to 137 species $(5.5 \%)$ of Yemen and 60 species $(5 \%)$ of Oman. However, there are about 152 undetermined specimens deposited at various herbaria in the Kingdom and Britain. The number of endemic species in Saudi Arabia probably goes even higher as and when the nomenclatural status of the undetermined specimens to be finalized. At present, most of the endemic species reported from the southwestern and northwestern highlands. It is probably due to these regions' rich variety of habitats and their affordable degree of environmental stability (Miller \& Nyberg, 1991).

\section{Study Area:}

Al-Baha region lies in the southwest of the kingdom of Saudi Arabia, located between the area of Makkah, and Asir, it is the smaller provinces of Saudi Arabia with a 
total area of 11000 square kilometers, surrounded by many of the cities where bordered to the north by the city of Taif, from the east is Bisha city, from the southern is Abha city, and to the West by the center of the Quanfonda. Al-Baha region has high plant vegetation, dense agricultural spaces. The six major cities of Al-Baha region are Beljarshy, Almandaq, and Almekhwah, in addition to Al-Baha city in the center of the province. The province comprises 31 administrative centers and has a population of 533,001. It is known for its forests, wildlife areas, valleys, and mountains that attract visitors from all parts of the kingdom and the Arabic Gulf area. Some of these areas are the forests of Raghdan, Ghomsan, Fayk, and Aljabal, and many other historical and archeological sites. It contains more than 53 forests. Al-Baha region is situated in the central foothills of the western mountains at an altitude of approximately $1899.68 \mathrm{~m}$ above sea level $\left(20^{\circ} 1771^{\prime} \mathrm{N} 04^{\circ} 2757^{\prime} \mathrm{E}\right)$ (Map 1). The climate in Al-Baha greatly affected by its varying geographic features. Generally, the climate in Al-Baha is mild with temperatures ranging between 12 to $23{ }^{\circ} \mathrm{C}\left(53.6\right.$ to $\left.73.4^{\circ} \mathrm{F}\right)$. Due to its location at 2,500 meters $(8,200 \mathrm{ft})$ above sea level, Al-Baha's climate is moderate in summer and cold in winter. The area attracts visitors looking for a moderate climate and pristine, scenic views. In the Tehama area of the province, which is down on the coast, the climate is hot in the summer and warm in the winter. Humidity ranges from 52\%-67\%. While in the mountainous region, which known as As-Sarah, the weather is cooler in summer and winter, (Yassin, et al., 2013). Rainfall in the mountainous region lies in the range of 229 to 581 millimeters ( 9 to 23 in). The average throughout the whole region is 100 to 250 millimeters (3.9 to 9.8 in) annually, (Abdul-Razzaq, 2001), (Table 1).

Map 1: Showing Al-Baha region of Saudi Arabia, (http://www.citiestips.com/city/AlBahaSaudiArabia)

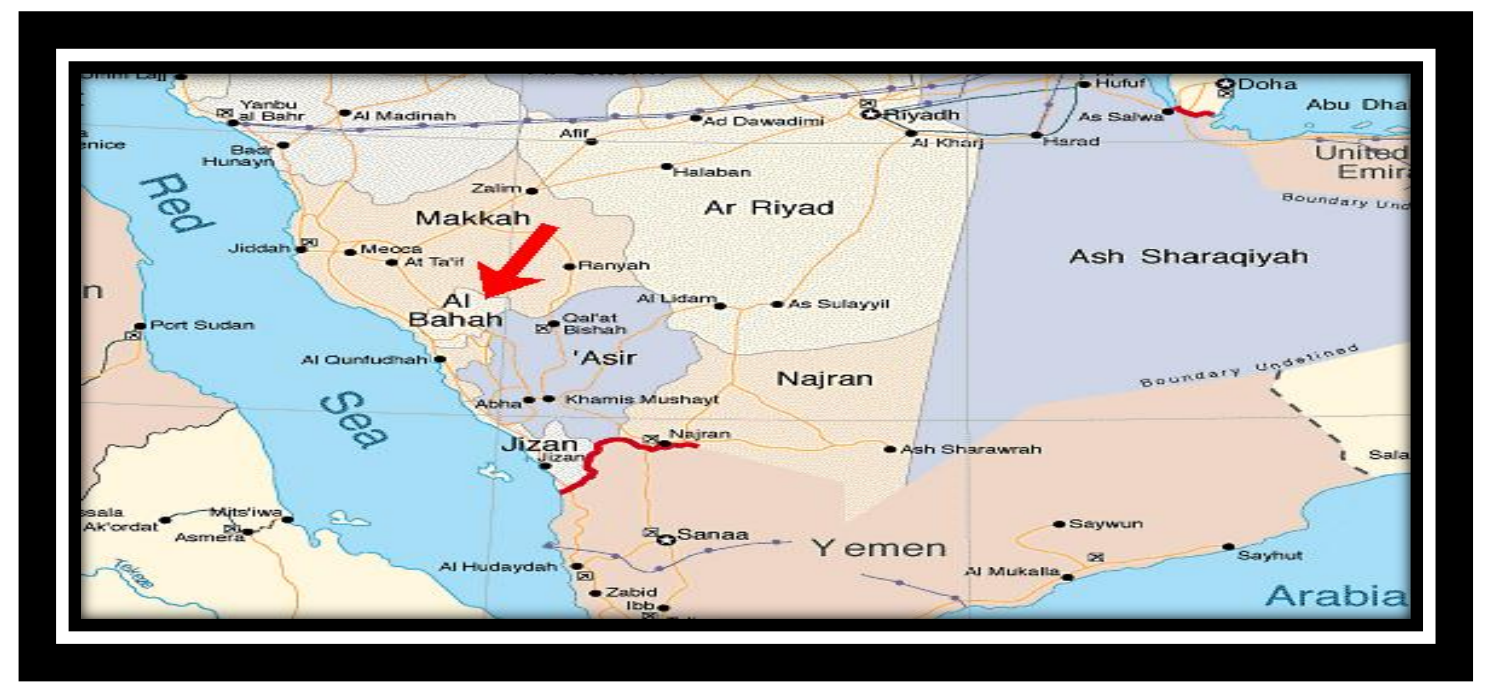


Table 1: Monthly variation in air temperature $\left({ }^{\circ} \mathrm{C}\right)$, relative humidity (percentage), and rainfall $\left(\mathrm{mm}\right.$ month $\left.^{\circ}\right)$ as recorded at Al-Baha region that located in the study area. The data are long-term average (Jeddah Regional Climate Center, KSA, 1985-2015).

\begin{tabular}{|c|c|c|c|c|c|c|c|c|c|c|c|c|c|}
\hline \multicolumn{14}{|c|}{ Climate data for Al-Baha region (1985-2015) } \\
\hline Month & Jan & Feb & Mar & $\underset{\mathbf{r}}{\mathbf{A p}}$ & $\begin{array}{c}\mathrm{Ma} \\
\mathbf{y}\end{array}$ & Jun & Jul & $\begin{array}{c}\mathrm{Au} \\
\mathrm{g}\end{array}$ & Sep & Oct & $\begin{array}{c}\text { No } \\
\mathbf{v}\end{array}$ & Dec & $\begin{array}{c}\text { Yea } \\
\mathbf{r}\end{array}$ \\
\hline Record high ${ }^{\circ} \mathrm{C}$ & 29 & 32 & 36 & 35 & 38 & 39 & 38 & 39 & 38 & 35 & 37 & 29 & 39 \\
\hline Average high ${ }^{\circ} \mathrm{C}$ & 22 & 24 & 26 & 29 & 32 & 35 & 35 & 35 & 33 & 29 & 26 & 23 & 29 \\
\hline Daily mean & 15 & 17 & 20 & 22 & 25 & 28 & 28 & 29 & 27 & 23 & 19 & 16 & 22 \\
\hline Average low ${ }^{\circ} \mathrm{C}$ & 9 & 11 & 13 & 16 & 19 & 22 & 22 & 22 & 20 & 16 & 13 & 10 & 16 \\
\hline Record low ${ }^{\circ} \mathrm{C}$ & 1.6 & 0.0 & 4.0 & 8.0 & $\begin{array}{c}12 . \\
2\end{array}$ & $\begin{array}{c}12 . \\
0\end{array}$ & $\begin{array}{c}15 . \\
8\end{array}$ & $\begin{array}{c}14 . \\
0\end{array}$ & $\begin{array}{c}15 . \\
0\end{array}$ & 8.5 & 5.8 & 2.0 & 0.0 \\
\hline $\begin{array}{l}\text { Average } \\
\text { precipitation } \mathbf{~ m m}\end{array}$ & 10.9 & 1.1 & 16.5 & $\begin{array}{c}36 . \\
3\end{array}$ & $\begin{array}{c}24 . \\
1\end{array}$ & 6.0 & $\begin{array}{c}10 . \\
2\end{array}$ & $\begin{array}{c}10 . \\
8\end{array}$ & 2.9 & 7.2 & 8.1 & 4.0 & $\begin{array}{c}138 . \\
1\end{array}$ \\
\hline $\begin{array}{l}\text { Average } \\
\text { precipitation days }\end{array}$ & 22 & 1.0 & 3.9 & 9.5 & 8.7 & 2.7 & 3.9 & 5.5 & 1.5 & 2.1 & 3.5 & 2.8 & 47.3 \\
\hline $\begin{array}{l}\text { Average relative } \\
\text { humidity }(\%)\end{array}$ & 55 & 48 & 46 & 45 & 35 & 25 & 27 & 28 & 25 & 30 & 46 & 53 & 39 \\
\hline
\end{tabular}

\section{Materials and Methods:}

The important sites in Al-Baha region (Mashnyia, Medhas Dams, Darak, and Elkhalab valleys) were visited and surveyed in 2017 and 2018. In each site, the following data were recorded: First and second dominant plant species, GPS data (global positioning system) and height above sea level, photography of all plant species, list of the annual, perennial, and biannual plant species. Collection samples of different plant species from most sites. The herbarium sheets of the recorded species kept in the Herbarium of Biology Department, Faculty of Science and Arts, Al-Baha University. Nomenclature according to (Boulos, 1983 and 1995), (Shaltout, 1997) and (Tackhlom, 1974). The potential and actual economic uses of the wild plant species assessed, on three bases:

- Field observations.

- Information collected from local inhabitants.

- Literature reviews and recording the origin of each plant species. 


\section{Results and Discussion}

The floristic composition of Mandaq province, Al-Baha was collected from four regions: Mashnia Dam (123 species), Medhas Dam (102 species), Darak Valley (103 species), and Elkhalab valley (84 species) (five sites for each). Plants of the database described using the following attributes; family name, botanical name, life form, common uses, origin (native), and locations of presence (Table 2). A total of 160 species of the vascular plants were recorded belonging to 52 families. Largest families (included the highest number of species) were Poaceae (22 species $=13.7 \%)$ and Asteraceae $(19$ species $=11.8 \%)$ followed by Fabaceae $(11$ species $=6.8 \%)$. Three families were represented by six species such as Amaranthaceae, Solanaceae, three families were represented by five species such as Borabinaceae and Moraceae. Three families represented by four species (Chenopodiaceae, Euphorbiaceae, and Malvaceae). While the families represented by three species were six such as Anacardiaceae, Resedaceae, and Rhamnaceae. There are ten plant families represented by only two species such as Aizoaceae, Aloaceae, and Apocynaceae. On the other hand, there are 23 families represented by only one species such as Cyperaceae, Myrtaceae, and Meliaceae (Figure 1).

According to life forms, the annual herbs had the highest contribution of the recorded plant species $(56$ species $=35 \%)$, followed by perennial herbs $(48$ species $=$ $30 \%)$, shrubs and shrublets $(26$ species $=16.25 \%)$, Trees $(23$ species $=14.37 \%)$, biennial herbs (five species $=3.12 \%$ ). On the other hand, the palms and cactus had the lowest contribution (one species for each) (Figure 2).

Figure 1: Survey of the recorded plant families in Al-mandq, Al-Baha (KSA).

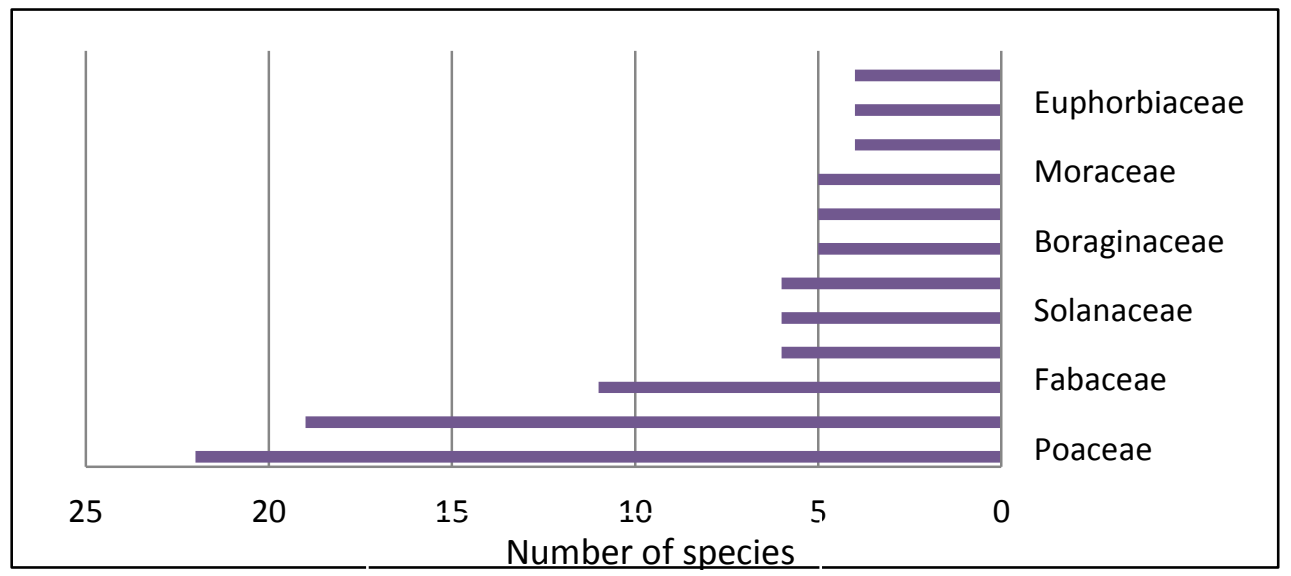




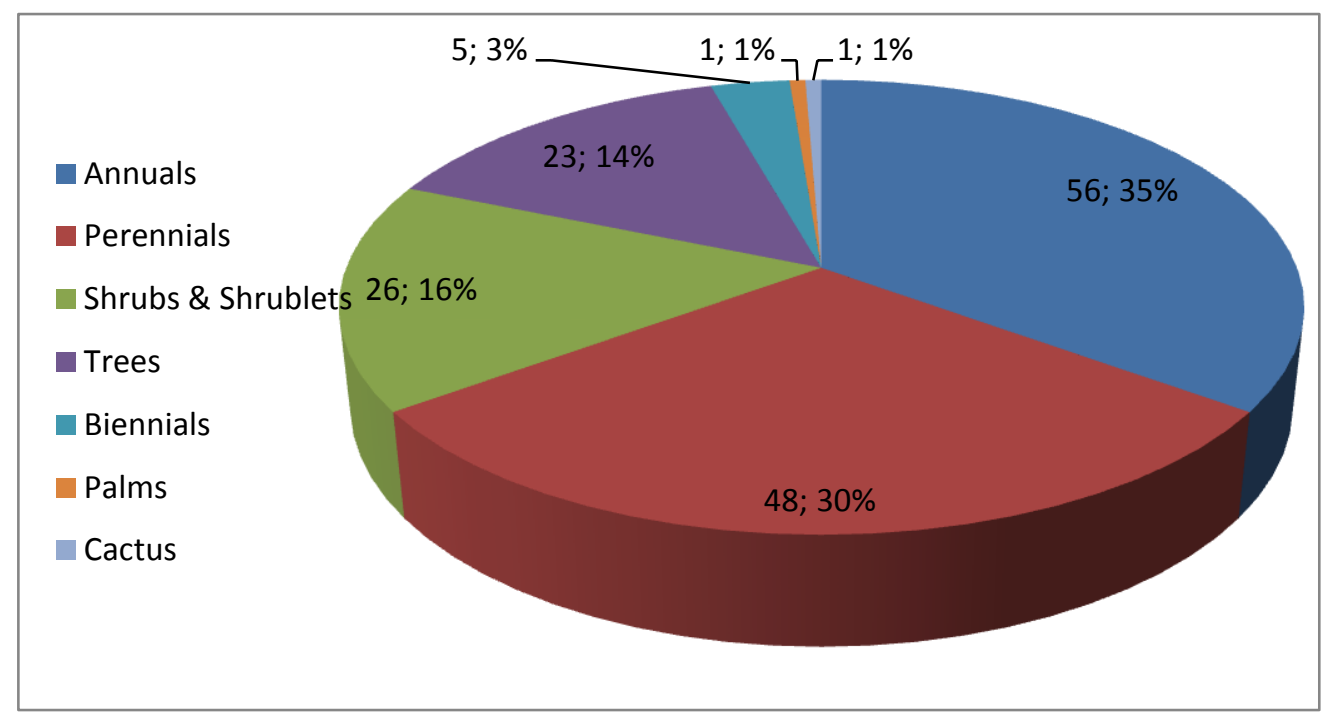

Figure 2: Classification of life forms of the recorded plant species in Almandq, AlBaha region, $(\mathrm{KSA})$.

Seventy-nine of total recorded plant species (49.37\%) have medicinal uses, Twenty-five of total recorded plant species $(15.62 \%)$ are edible, and while twenty of total recorded plant species (12.5-\%) are used as ornamental. On the other hand, there are fifteen of total recorded plant species $(9.37 \%)$ are foliage, thirteen of total plant species $(8.12 \%)$ are used as economic, finally eight of total recorded plant species (5\%) are has biological uses (Figure 3).

Figure 3: Classification of the recorded plant species of Al-Baha region according to common uses.

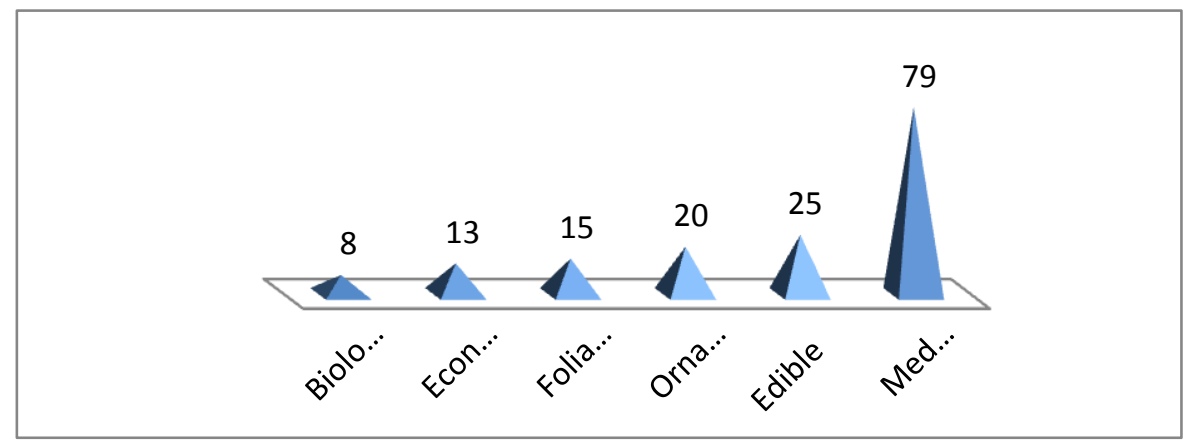




\section{Table 2: Floristic composition of plant species recorded at different locations of Almandq Province, Al-Baha Region}

(KSA).

\begin{tabular}{|c|c|c|c|c|c|c|c|c|}
\hline \multirow{2}{*}{ Family } & \multirow{2}{*}{ Botanical Name } & \multirow{2}{*}{$\begin{array}{l}\text { Life } \\
\text { form }\end{array}$} & \multirow{2}{*}{ C.U. } & \multirow{2}{*}{ Origin (Native) } & \multicolumn{4}{|c|}{ Locations } \\
\hline & & & & & Mas. & Med. & Dha. & Elk \\
\hline Acanthaceae & Blepharis ciliaris (L.) B. L. Burtt & Perennial & M. & Arab. Pen. & + & + & + & - \\
\hline Aizoaceae & Aizoon canariense L. & Annual & Orn. & Maur. \& Mali & + & + & - & + \\
\hline Aizoaceae & Gisekia pharnaceoides L. & Annual & M. & India & + & + & - & - \\
\hline Aloaceae & $\begin{array}{l}\text { Aloe pseudorubroviolacea } \\
\text { Lavranos \& Collen }\end{array}$ & Perennial & M. & KSA & - & - & + & + \\
\hline Aloaceae & Aloe vera (L.) Burm.f. & Perennial & M. & Arab. Pen. & + & + & + & - \\
\hline Amaranthaceae & Achyranthes aspera L. & Perennial & M. & Tropics & - & + & + & - \\
\hline Amaranthaceae & Aerva javanica (Burm.f.) Shult & Perennial & M. & W. \& S. Asia & - & + & + & - \\
\hline Amaranthaceae & Alternanthera pungens Kunth & Annual & M. & Am. & + & - & - & - \\
\hline Amaranthaceae & Amaranthus graecizans L. & Annual & Ed. & E. \& C. Asia to India & - & - & + & + \\
\hline Amaranthaceae & Amaranthus tricolor L. & Annual & Orn. & S. Am. & + & - & + & - \\
\hline Amaranthaceae & Amaranthus viridis L. & Annual & M. & India & - & + & + & + \\
\hline Anacardiaceae & Pistacia atlantica L. & Tree & Orn. & N. Afr. & - & - & + & + \\
\hline Anacardiaceae & $\begin{array}{l}\text { Pistacia falcata Beccari ex } \\
\text { Martelli }\end{array}$ & Tree & Orn. & $\begin{array}{l}\text { KSA, Oman, Yemen } \\
\text { \& Afr. }\end{array}$ & - & + & + & - \\
\hline Anacardiaceae & Rhus glabra L. & Shrub & M. & N. Am. & + & - & + & + \\
\hline Apiaceae & Anethum graveolens L. & Annual & M. & Asia \& Med. & - & + & + & - \\
\hline Apocynaceae & Carissa spinarum L. & Shrub & Ed. & Afr., S. Asia, \& Aus. & + & - & - & - \\
\hline Apocynaceae & $\begin{array}{l}\text { Gomphocarpus fruticosus (L.) } \\
\text {.T.Aiton }\end{array}$ & Shrub & M. & S. Afr. & - & + & - & + \\
\hline Arecaceae & Phoenix dactylifera L. & Palm & Eco. & S. Asia \& N. Afr. & + & + & + & - \\
\hline Asclepiadaceae & Calotropis procera (Aiton) R.Br. & Shrub & M. & $\begin{array}{l}\text { N. Afr., W. \& S. } \\
\text { Asia }\end{array}$ & + & + & + & - \\
\hline Asclepiadaceae & $\begin{array}{l}\text { Leptadenia pyrotechnica } \\
\text { (Forssk.) Decne. }\end{array}$ & Perennial & Fol. & Afr. \& India & - & - & + & + \\
\hline Asphodelaceae & Asphodelus tenuifolius Cav. & Annual & Eco. & $\begin{array}{l}\text { Med., Asia \& Mas. } \\
\text { Is. }\end{array}$ & + & - & + & - \\
\hline Asteraceae & $\begin{array}{l}\text { Achillea fragrantissima (Forssk.) } \\
\text { Sch.Bip. }\end{array}$ & Shrub & M. & $\begin{array}{l}\text { Eu., t. Asia \& N. } \\
\text { Am. }\end{array}$ & - & + & - & + \\
\hline Asteraceae & Anthemis cotula L. & Annual & M. & Med. & - & - & + & + \\
\hline Asteraceae & Anvillea garcinii (Burm.f.) DC. & Shrub & M. & $\begin{array}{l}\text { N. Afr., Mid. East, \& } \\
\text { Arab. Pen. }\end{array}$ & - & + & - & + \\
\hline Asteraceae & $\begin{array}{l}\text { Artemisia abyssinica Sch.Bip. ex } \\
\text { A.Rich }\end{array}$ & Shrub & M. & Eurasia \& N. Afr. & + & + & - & + \\
\hline Asteraceae & Artemisia vulgaris L. & Perennial & M. & Eu. \& N. Am. & - & + & - & - \\
\hline Asteraceae & $\begin{array}{l}\text { Atractylis carduus (Forssk.) } \\
\text { Christ }\end{array}$ & Annual & M. & Greek Is. of Crete & + & - & + & - \\
\hline Asteraceae & Bidens pilosa $\mathrm{L}$. & Annual & M. & Am. & + & + & - & - \\
\hline Asteraceae & Calendula arvensis L. & Annual & Orn. & C. \& S. Eu. & + & + & + & - \\
\hline Asteraceae & Centaurea pseudosinaica Czerep. & Annual & M. & Calif. & - & + & - & + \\
\hline Asteraceae & Conyza bonariensis (S.Moore) & Perennial & M. & Tr. \& W. Reg. & - & + & + & + \\
\hline
\end{tabular}




\begin{tabular}{|c|c|c|c|c|c|c|c|c|}
\hline & Cufod. & & & & & & & \\
\hline Asteraceae & Echinops spinosissimus Turra & Perennial & Fol. & $\begin{array}{l}\text { S. Eu.,N. Afr., \& S. } \\
\text { Asia }\end{array}$ & + & + & & \\
\hline Asteraceae & $\begin{array}{l}\text { Flaveria trinervia (Spreng.) } \\
\text { C.Moh. }\end{array}$ & Annual & M. & Aus. & + & - & - & - \\
\hline Asteraceae & $\begin{array}{l}\text { Imperata cylindrical (L.) P. } \\
\text { Beauv. }\end{array}$ & Perennial & M. & E. \& S. Asia & + & + & + & + \\
\hline Asteraceae & Onopordum acaulon L. & Biennial & M. & $\begin{array}{l}\text { S. Eu., N. Afr., \& } \\
\text { Can. Is. }\end{array}$ & - & + & - & + \\
\hline Asteraceae & Pulicaria vulgaris Gaertn. & Shrub let & M. & Alb., Alg. \& Bos. & - & + & + & + \\
\hline Asteraceae & Reichardia tingitana (L.) Roth & Annual & M. & Afr. & - & + & - & + \\
\hline Asteraceae & Senecio vulgaris L & Annual & M. & Eu. & + & + & + & + \\
\hline Asteraceae & Sonchus oleraceus L. & Annual & M. & Eu., \& W. Asia & + & + & + & + \\
\hline Asteraceae & Xanthium strumarium L. & Perennial & M. & N. Am. & + & + & - & + \\
\hline Boraginaceae & Alkana orientalis (L.) Boiss. & Perennial & Eco. & S. Eu. & + & - & - & - \\
\hline Boraginaceae & Anchusa arvensis (L.) M.Bieb. & Annual & Ed. & Eu. & - & + & - & - \\
\hline Boraginaceae & Heliotropium arborescens L & Shrub & M. & Peru & + & - & - & - \\
\hline Boraginaceae & $\begin{array}{l}\text { Heliotropium ramosissimum } \\
\text { (Lehm.) DC. }\end{array}$ & Perennial & M. & Arab. Pen. \& Eu. & + & + & - & - \\
\hline Boraginaceae & $\begin{array}{l}\text { Moltkiopsis ciliata (Forssk.) } \\
\text { I.M.Johnston }\end{array}$ & Annual & Fol. & Afr. \& Asia & + & - & + & - \\
\hline Brassicaceae & Brassica tournefortii Gouan & Annual & Ed. & Calif. & + & + & - & - \\
\hline Brassicaceae & $\begin{array}{l}\text { Capsella bursa-pastoris (L.) } \\
\text { Medik. }\end{array}$ & Annual & Fol. & E. Eu. \& Asia & + & + & - & + \\
\hline Brassicaceae & Diplotaxis erucoides (L.) DC. & Annual & Ed. & W. Med. & + & - & + & - \\
\hline Brassicaceae & Farsetia stylosa R. Br & Annual & Fol. & Afr. & + & + & - & + \\
\hline Brassicaceae & Sisymbrium irio L. & Annual & M. & Med. \& Britain & + & + & + & - \\
\hline Cactaceae & Opuntia ficus-indica (L.) Mill. & Cactus & Ed. & Mexico & + & + & + & + \\
\hline Cannabaceae & Celtis africana Burm. f. & Tree & Ed. & S. Afr. \& Eth. & - & - & + & - \\
\hline Capparaceae & Boscia angustifolia A. Rich. & Shrub & M. & Afr. \& semi-arid Tr. & - & + & + & + \\
\hline Capparaceae & Capparis spinosa L. & Shrub & M. & Med. & + & - & + & - \\
\hline Chenopodiaceae & Bassia indica (Wight) A. J. Scott & Perennial & Fol. & Arab. Pen.\& W. Asia & + & + & + & + \\
\hline Chenopodiaceae & Bassia muricata (L.) Aschers. & Perennial & M. & W. Med.\& E. Asia & + & - & + & - \\
\hline Chenopodiaceae & Chenopodium album L. & Annual & Ed. & Eu. & + & - & + & + \\
\hline Chenopodiaceae & Chenopodium murale L. & Annual & M. & Eu. \& Asia & + & + & + & + \\
\hline Convolvulaceae & Convolvulus arvensis L. & Perennial & M. & Eu. \& Asia & + & + & + & - \\
\hline Cucurbitaceae & Citrllus colocynthis (L.) Schard. & Perennial & M. & Med \& Asia & - & - & - & + \\
\hline Cupressaceae & Cupressus sempervirens L. & Tree & Orn. & E. Med., \& N. Lib. & + & + & - & + \\
\hline Cupressaceae & Juniperus phoenicea L. & Tree & Eco. & W. KSA \& Med. & + & + & + & + \\
\hline Cupressaceae & $\begin{array}{l}\text { Juniperus procera Hochst. ex } \\
\text { Endl. }\end{array}$ & Tree & Eco. & $\begin{array}{l}\text { Mou. Afr. \& Arab. } \\
\text { Pen. }\end{array}$ & + & + & + & + \\
\hline Caryophyllaceae & Gymnocarpos decandrus Forssk. & Perennial & M. & N. Afr. \& Asia & - & - & + & + \\
\hline Cyperaceae & Cyperus rotundus L. & Perennial & Fol. & $\begin{array}{l}\text { Afr. S. Eu. \& S. } \\
\text { Asia }\end{array}$ & + & - & - & + \\
\hline Ericaceae & Erica arborea L. & Shrub & Eco. & Afr. & + & - & + & - \\
\hline Euphorbiaceae & Euphorbia helioscopia L. & Annual & M. & Eu., Afr. \& Asia & + & - & + & - \\
\hline Euphorbiaceae & Euphorbia hirta L. & Annual & M. & India & + & - & + & - \\
\hline Euphorbiaceae & Euphorbia peplus L. & Annual & M. & Eu., N. Afr., and W. & + & - & + & - \\
\hline
\end{tabular}




\begin{tabular}{|c|c|c|c|c|c|c|c|c|}
\hline & & & & Asia & & & & \\
\hline Euphorbiaceae & Ricinus communis L. & Shrub & M. & Tr. Afr. & + & - & + & + \\
\hline Fabaceae & Acacia ehrenbergiana Hayne. & Tree & Fol. & E. Afr. \& Arab. Pen. & + & + & - & - \\
\hline Fabaceae & Acacia etbaica Schweinf. & Shrub & Eco. & Afr. \& Aus. & - & + & + & - \\
\hline Fabaceae & Acacia gerrardii Benth. & Tree & Eco. & Afr. \& W. Asia & + & + & - & + \\
\hline Fabaceae & Acacia seyal Del. & Tree & M. & Afr. & + & - & + & + \\
\hline Fabaceae & Acacia tortilis (Forssk.) Hayne & Tree & M. & $\begin{array}{l}\text { Afr. (Sudan) \& Mid. } \\
\text { East }\end{array}$ & - & + & - & + \\
\hline Fabaceae & Alhagi maurorum Medik. & Perennial & M. & KSA, Eg. \& India & + & - & + & - \\
\hline Fabaceae & Anagyris foetida L. & Tree & Bio. & France & + & - & - & - \\
\hline Fabaceae & Astragalus corrugatus Bertol. & Perennial & M. & None & + & + & - & - \\
\hline Fabaceae & Indigofera tinctoria L. & Biennial & M. & Asia \& Afr. & - & + & - & + \\
\hline Fabaceae & Melilotus indicus (L.) All & Annual & Ed. & Asia, Eu. \& Asia & + & + & + & - \\
\hline Fabaceae & Trigonella stellata Forssk. & Perennial & M. & W. Asia & + & - & - & + \\
\hline Geraniaceae & Erodium cicutarium (L.) L'Hér. & Annual & Ed. & Afr. \& Asia & + & + & - & - \\
\hline Geraniaceae & Geranium ocellatum Cambess. & Annual & M. & N. Afr. to E. Asia & + & - & + & + \\
\hline Lamiaceae & Lavandula pubescens Decne. & Perennial & Orn. & Eg., Er. \& Asia & + & + & + & + \\
\hline Lamiaceae & Mentha longifolia (L.) Huds. & Perennial & M. & Eu., Afr. \& Asia & + & + & + & + \\
\hline Lamiaceae & Rosmarinus officinalis L. & Perennial & M. & Med. & - & - & + & - \\
\hline Malvaceae & $\begin{array}{l}\text { Abutilon fruticosum Guill. \& } \\
\text { Perr. }\end{array}$ & Perennial & Orn. & Asia, Afr. \& India & + & + & - & - \\
\hline Malvaceae & Althaea ludwigii L. & Perennial & M. & Asia, Afr. \& Eu. & - & - & - & + \\
\hline Malvaceae & Malva parviflora L. & Annual & Ed. & Afr. \& Eu. & + & + & + & - \\
\hline Malvaceae & Malva verticillata $\mathrm{L}$. & Biennial & Ed. & E. Asia \& Ch. & + & - & + & + \\
\hline Meliaceae & Azadiracchta indica L. & Tree & Bio. & India, Bur. \& Ban. & + & + & + & + \\
\hline Moraceae & Ficus carica L. & Tree & Ed. & Mid. East \& W. Asia & + & + & + & \\
\hline Moraceae & Ficus cordata Thunb. & Tree & Orn. & Arab. Pen. \& Afr. & + & - & + & + \\
\hline Moraceae & Ficus ingens (Miq.) Miq. & Tree & Ed. & Arab. Pen. \& T. Afr. & + & - & + & - \\
\hline Moraceae & Ficus palmata Forssk. & Tree & Ed. & Arab. Pen. \& N. Afr. & + & - & + & + \\
\hline Moraceae & Ficus salicifolia Vahl. & Tree & Orn. & Arab. Pen. \& Afr. & + & + & + & - \\
\hline Myricaceae & Morella cordifolia (L.) Killick & Shrub & Orn. & S. Africa & + & + & - & - \\
\hline Myrtaceae & Eucalyptus globulus Labill & Tree & M. & Aus. & + & + & + & + \\
\hline Oleaceae & $\begin{array}{l}\text { Olea europaea L. subsp. africana } \\
\text { (Mill.) P. S. Green }\end{array}$ & Tree & Ed. & $\begin{array}{l}\text { Afr., Arab. Pen. \& } \\
\text { Mas. Is. }\end{array}$ & + & + & + & + \\
\hline Oxalidaceae & Oxalis corniculata L. & Perennial & Ed. & None & + & - & + & + \\
\hline Papaveraceae & Argemone mexicana L & Annual & M. & W. Am. \& Mexico & + & + & + & + \\
\hline Papaveraceae & Argemone ochroleuca Sweet. & Annual & M. & T. Am. & + & + & + & + \\
\hline Plantaginaceae & Plantago ciliata Desf. & Perennial & M. & Aus., Asia, \& Eu. & + & - & + & + \\
\hline Plantaginaceae & Plantago major L. & Perennial & Orn. & N. \& C. Asia & + & + & - & + \\
\hline Poaceae & Aristida adscensionis L. & Perennial & Bio. & T. Am. & - & + & + & + \\
\hline Poaceae & Arundo donax L. & Perennial & M. & $\begin{array}{l}\text { Med., Arab. Pen. \& } \\
\text { Calf. }\end{array}$ & + & - & + & - \\
\hline Poaceae & Avena fatua L. & Annual & M. & Eurasia & + & + & + & + \\
\hline Poaceae & Avena sativa L. & Annual & Ed. & Asia, Med. \& N. Afr. & + & - & + & - \\
\hline Poaceae & Cenchrus ciliaris L. & Perennial & Fol. & $\begin{array}{l}\text { S. Asia, S. Eu. \& } \\
\text { Afr. }\end{array}$ & + & + & + & - \\
\hline Poaceae & $\begin{array}{l}\text { Centropodia fragilis (Guinet } \& \\
\text { Sauvage) Cope }\end{array}$ & Perennial & Fol. & T. Asia \& Afr. & + & + & - & + \\
\hline Poaceae & Chloris gayana Kunth & Perennial & Fol. & Afr. & + & + & - & + \\
\hline
\end{tabular}




\begin{tabular}{|c|c|c|c|c|c|c|c|c|}
\hline Poaceae & Cynodon dactylon (L.) Pers. & Perennial & Orn. & Mid. East & + & + & + & + \\
\hline Poaceae & Digitaria ciliaris (Retz.) Koeler & Annual & Bio. & Asia & + & - & + & - \\
\hline Poaceae & Eragrostis minor Host. & Annual & Bio. & None & + & - & - & - \\
\hline Poaceae & $\begin{array}{l}\text { Hordeum murinum L. ssp. } \\
\text { glaucum (Steud.) Tzvelev }\end{array}$ & Annual & Fol. & Eu.,N Afr. \&T. Asia & + & + & + & - \\
\hline Poaceae & Hyparrhenia hirta (L.) Stapf & Perennial & Fol. & Afr. \& Eurasia & - & + & - & - \\
\hline Poaceae & Imperata cylindrica (L.) Raeus. & Perennial & Eco. & $\begin{array}{l}\text { E.S. Asia, India, } \\
\text { Aus. E \& S. Afr. }\end{array}$ & + & + & + & + \\
\hline Poaceae & Lolium multiflorum Lam. & Biennial & Bio. & $\begin{array}{l}\text { C., S. Eu. ,W. Afr. \& } \\
\text { S. Asia }\end{array}$ & - & + & - & + \\
\hline Poaceae & Lolium rigidum Gaud. & Annual & Fol. & $\begin{array}{l}\text { S. Eu., N. Afr., Mid. } \\
\text { East \& India }\end{array}$ & + & + & + & + \\
\hline Poaceae & Panicum turgidum Forssk. & Perennial & Eco. & Afr. \& Asia & + & + & + & + \\
\hline Poaceae & $\begin{array}{l}\text { Pennisetum setaceum (Forssk.) } \\
\text { Chiov. }\end{array}$ & Annual & Orn. & $\begin{array}{l}\text { E.,T. Afr. ,Mid. East } \\
\text { \& S. Asia }\end{array}$ & + & + & + & + \\
\hline Poaceae & $\begin{array}{l}\text { Polypogon monspeliensis (L.) } \\
\text { Desf. }\end{array}$ & Annual & M. & S. Eu. & + & + & + & - \\
\hline Poaceae & Schismus arabicus Nees & Annual & Fol. & S. Asia & + & - & + & + \\
\hline Poaceae & Setaria viridis (L.) P. Beauv. & Annual & M. & Eurasia & + & + & + & + \\
\hline Poaceae & Sorghum halepense (L.) Pers. & Perennial & M. & Med. \& W. Asia & + & + & - & - \\
\hline Poaceae & Themeda triandra Forssk. & Perennial & M. & Afr., Aus., \& Pac. & + & + & + & - \\
\hline Polygalaceae & $\begin{array}{l}\text { Polygala abyssinica R. Br. ex } \\
\text { Fresen. }\end{array}$ & Shrub & Orn. & Afr. \& Afg. & + & - & - & - \\
\hline Polygonaceae & Emex spinosa (L.) Campd. & Annual & Bio. & Asia, Eu. \& N. Am. & + & + & - & + \\
\hline Polygonaceae & Rumex vesicarius L. & Annual & Ed. & S. Eu. \& N. Afr. & + & + & + & + \\
\hline Portulacaceae & Portulaca oleracea L. & Annual & Ed. & $\begin{array}{l}\text { N. Afr., S. Eu., Mid. } \\
\text { East \& India }\end{array}$ & + & + & + & - \\
\hline Primulaceae & Anagallis arvensis L. & Annual & Orn. & $\begin{array}{l}\text { Eu., W. Asia \& N. } \\
\text { Afr. }\end{array}$ & - & - & + & + \\
\hline Resedaceae & $\begin{array}{l}\text { Caylusea hexagyna (Forssk.) M. } \\
\text { L. Green }\end{array}$ & Annual & Bio. & Afr. \& India & + & + & - & - \\
\hline Resedaceae & $\begin{array}{l}\text { Ochradenus arabicus Chaudhary, } \\
\text { Hillc. \& A.G.Mill. }\end{array}$ & Shrub & M. & Arab. Pen. \& S .Asia & + & - & + & + \\
\hline Resedaceae & Ochradenus baccatus Delile & Shrub & M. & Arab. Pen. & + & + & - & + \\
\hline Rhamnaceae & Rhamnus lycioides L. & Shrub & Ed. & E. Asia \& N. Am. & - & + & + & - \\
\hline Rhamnaceae & $\begin{array}{l}\text { Sageretia thea (Osbeck) } \\
\text { M.C.Johnst. }\end{array}$ & Shrub & Ed. & N. Afr. \& Arab. Pen. & + & + & + & + \\
\hline Rhamnaceae & Ziziphus spina-christi (L.) Desf. & Tree & Ed. & $\begin{array}{l}\text { N.,T. Afr. \&, S., W. } \\
\text { Asia }\end{array}$ & - & + & - & + \\
\hline Rosaceae & Crataegus $\times$ sinaica Boiss. & Tree & Ed. & Eu., Asia \& N. Am. & + & - & + & - \\
\hline Rosaceae & Prunus dulcis (Mill.) D. A. Webb & Tree & Ed. & W. Asia \& E. Med. & + & + & - & + \\
\hline Salvadoraceae & Salvadora persica L. & Shrub & Eco. & $\begin{array}{l}\text { T. Afr., Arab. Pen. \& } \\
\text { India }\end{array}$ & + & - & + & - \\
\hline Sapindaceae & Dodonaea viscosa (L.) Jacq. & Shrub & M. & $\begin{array}{l}\text { Afr. , Asia, Aus. \& } \\
\text { Am. }\end{array}$ & + & + & + & + \\
\hline Scrophulariaceae & Verbascum sinaiticum Benth & biennial & Orn. & Eu. \& Asia & + & + & - & - \\
\hline Solanaceae & Datura innoxia Mill. & Perennial & M. & S. Am. & + & - & + & + \\
\hline Solanaceae & Datura stramonium L. & Annual & M. & N. Am. & - & + & - & + \\
\hline Solanaceae & Lycium shawii Roem. \& Schult & Shrub & M. & Aus. & + & - & - & - \\
\hline Solanaceae & Nicotiana glauca Graham & Shrub & Eco. & S. Am. & + & + & + & + \\
\hline Solanaceae & Solanum forsskalii Dunal & Shrublet & M. & T. Asia \& Aus. & + & + & + & + \\
\hline Solanaceae & Solanum villosum Miller & Annual & M. & Eu., W Asia, N. & + & + & + & - \\
\hline
\end{tabular}




\begin{tabular}{|c|c|c|c|c|c|c|c|c|}
\hline & & & & Afr.,\& N. Am. & & & & \\
\hline Solanaceae & Withania somnifera (L.) Dunal & Perennial & M. & $\begin{array}{l}\text { N. Afr., W., S. Asia, } \\
\text { S. Eu., Med. \& Can. } \\
\text { Is }\end{array}$ & + & 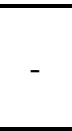 & + & \\
\hline Tamaricaceae & Tamarix aphylla (L.) Karst. & Tree & M. & $\begin{array}{l}\text { Afr., Mid. East, \& W. } \\
\text { S. Asia }\end{array}$ & + & + & + & + \\
\hline Typhaceae & Typha elephantine Roxb & Perennial & M. & Asia \& Afr. & + & - & - & - \\
\hline Urticaceae & Forsskaolea tenacissima L. & Annual & Eco. & $\begin{array}{l}\text { N. Afr., S. Eu. \& W. } \\
\text { Asia }\end{array}$ & + & + & - & + \\
\hline Urticaceae & Parietaria alsinifolia Delile & Annual & M. & Eurasia \& Afr. & + & + & - & - \\
\hline Urticaceae & Urtica dioica L. & Perennial & M. & $\begin{array}{l}\text { Eu., Asia, N. Afr. \& } \\
\text { N. Am. }\end{array}$ & - & + & + & + \\
\hline Zygophyllaceae & $\begin{array}{l}\text { Fagonia boveana (Hadidi) Hadidi } \\
\text { \& Graf }\end{array}$ & Shrublet & M. & India \& Eg. & + & + & - & - \\
\hline Zygophyllaceae & Fagonia indica Burm. f. & Perennial & M. & India \& Afr. & + & + & + & - \\
\hline Zygophyllaceae & Peganum harmala L. & Perennial & M. & S. Asia \& Mid. East & + & - & + & - \\
\hline Zygophyllaceae & Seetzenia lanata (Willd.) Bullock & Annual & Orn. & Asia, Afr. \& Aus. & + & - & + & - \\
\hline Zygophyllaceae & Tribulus arabicus Hosn. & Perennial & Orn. & T. Asia \& S. Afr. & + & + & + & - \\
\hline $\begin{array}{l}\text { Zygophyllaceae } \\
\text { (Total No.): } 52\end{array}$ & $\begin{array}{l}\text { Tribulus terrestris L. } \\
160\end{array}$ & $\begin{array}{c}\text { Annual } \\
8\end{array}$ & $\begin{array}{c}\text { Orn. } \\
6\end{array}$ & $\begin{array}{l}\text { S. Asia, S. Eu. \& } \\
\text { Afr. } \\
\qquad 31\end{array}$ & $\begin{array}{c}+ \\
123\end{array}$ & $\begin{array}{c}- \\
102\end{array}$ & $\begin{array}{c}+ \\
103 \\
\end{array}$ & $\begin{array}{l}+ \\
84\end{array}$ \\
\hline
\end{tabular}

Most of the recorded plant species have at least one aspect of potential or actual economic uses (Table 2: Appendix).

\section{Abbreviations:}

Afghanistan $=$ Afg. Africa $=$ Afr. Albania $=$ Albn., Algeria $=$ Alg. America $=$ Am. Arabian Peninsula $=$ Arab. Pen. Australia $=$ Aus. Bangladesh $=$ Bangl. Biological $=$ Bio Bosnia $=$ Bosn. Burma $=$ Bur. California $=$ Calif. Canary $=$ Canr. Central $=$ C. China $=$ Ch., Common Uses $=$ C.U. East $=$ E. Edible $=$ Ed. Economic $=$ Eco. Egypt $=$ Egy. Eritrea $=$ Eri. Ethiopic $=$ Eth. Europe $=$ Eu. France $=$ Fra. Foliage $=$ Fol. Kingdom Saudi Arabia $=$ K. S. A. Libya $=$ Lib. Mascarene Islands $=$ Mas. Is. Mauritania and Mali = Maur. \& Mali. Medicinal $=$ M. Mediterranean $=$ Med. Middle $=$ Mid. Mountains $=$ Mou. North $=$ N. Ornamental $=$ Orn., Pacific $=$ Pac. South $=$ S. Regions $=$ Regns. Tropic $=$ Tr. West $=$ W. $(-)=$ Absent and $(+)=$ Present. 
Unfortunately; the standard reference, Migahid's Flora of Saudi Arabia, gives no indication of the origins of the species it contains, and a number, including, for example, Azadirachta indica, Cupressus sempervirens, Eucalyptus camaldulensis, Morus nigra, Gossypium arboreum, Ricinus communis, Clitoria ternatea, Cynodon dactylon, Arundo donax and Phragmites australis; seem certainlly to be introduced from neighbouring regions in the historic or very recent past (Colleqette, 1985). Indeed, some species recently introduced, such as Prosopis juliflora, are so well adapted to local conditions that it must be only a matter of time before they spontaneously spread into suitable natural locations throughout the country, (Mandaville, 1990). Figure 4: showing the origin of different plant species in Almandq region, Al-Baha, KSA.

Figure 4: Distribution according to the origin of the recorded plant species in Almandq Province, Al-Baha region, (KSA).

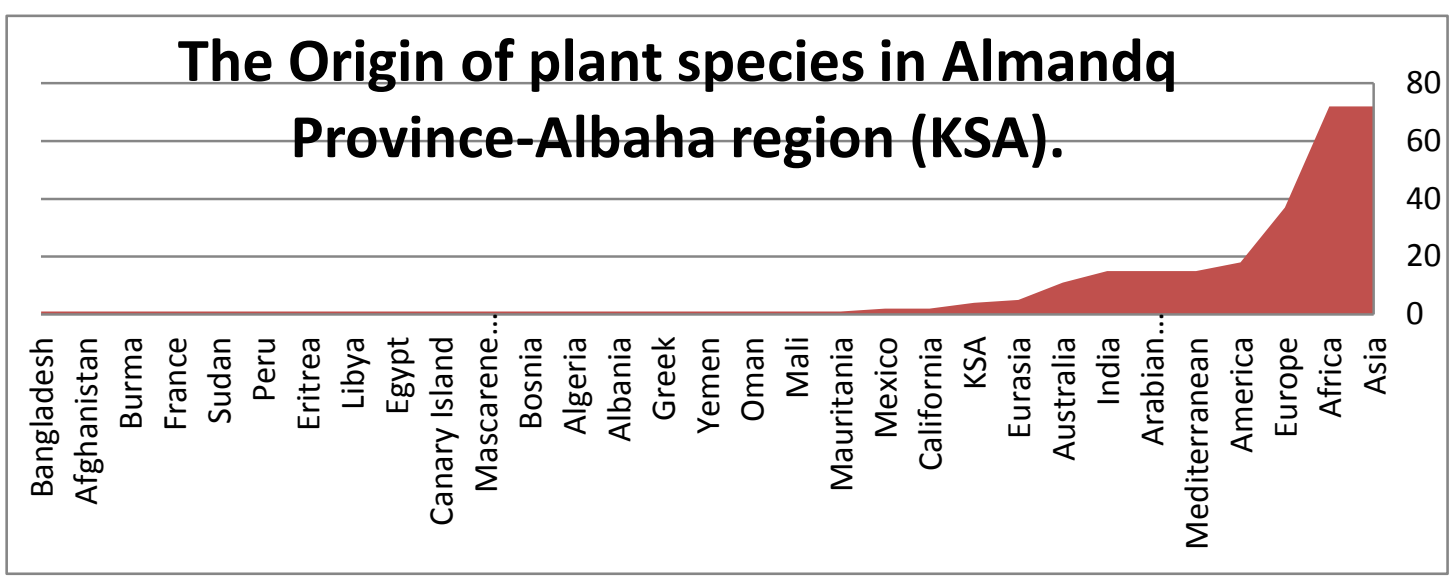

To conserve the different habitats in KSA make a sketch of the required area to be conserved noting all existing plants, animals, building, utilities, and pathways. Some trees be planted too close to buildings or have grown much larger than the previous owner envisioned. Some species may be of little wildlife value and may not be particularly attractive. Once we have identified existing plants to saved, exploring options for plants that work well these species can be started (Allen \& Gabrielle 2004).

One of steps to conserve our habitats, adding trees, shrubs, flowers, and ground covers to our plan. Not all the planting needs to be done at once. If money or time is limited, consider it a work in progress (Schultz \& Whitney, 1986). Therefore, to protect useful plants and ensure availability, the public should be educated to the importance of the plants and all possible avenues used to encourage the public to become acquainted with their medicinal uses and to cultivate the plants on the farms and in gardens and flowers pots for therapeutic uses. The use of the plants would undoubtedly minimize the cost of treatment and limit side or toxic effects of orthodox medicines that are currently being used (Sangwan, 2011). It is an open database of floristic composition with a broadspectrum attributes and may be useful for the scientific community for getting quick information about the medicinal, edible, ornamental, foliage, economic, and biological plants in Al-Baha region (KSA). 


\section{Acknowledgment:}

This paper is a part of research project entitled: "Phytochemical screening of some Saudi plant from AL-Mandaq province, AL-Baha region", supported by the government of Kingdom of Saudi Arabia, Ministry of Education, Al Baha University through project number: (100/ 1438). This grant greatly appreciated. We thank Professor Dr. A. Al Hussain the Vice Chancellor of the university and Dr. Naif, A. Addossary, Deanship of Scientific Research, Al Baha University. Appreciations also extended to Dr. Khalid bin Salim, dean faculty of sciences and arts, al Mandaq and to reviewers who provided helpful comments to improve the first version of this manuscript.

\section{REFERENCES}

Abdul-Razzaq, H. (2001) . "Social Functions of Weekly Markets in Al-Baha."Journal of the Social Sciences. ISSN 0253-1097. Published by Academic Publication Council - Kuwait University. Volume 29 (2).

Alfarhan A., Chaudhary S., Thomas J. (1998) . Notes on the flora of Saudi Arabia (Third edition). J. King Saud Univ., 10(1):31-40.

Allen D. \& Gabrielle H. (2004) : Medicinal Plants in Folk Tradition. An Ethnobotany of Britain \& Ireland. Portland / Cambridge, Timber Press.

Anonymous, (2000) . The ladybird discovery encyclopedia of the natural world.Ladybird Book limited MCMXCVII. Singapore, pp. 116-132.

Babaloa, O.(1988). Antibacterial properties of crude extracts of the sap and bark of Pycanthus angulata. Nigerian J. Microbial, 8: 4-11.

Boulos L., (1983). Medicinal Plants of North Africa. Reference Publication, Inc., Algonas Michigan.

Boulos L., (1995). Flora of Egypt. Vol. 1, Al-Hadara Publishing, Cairo, Egypt.

Colleqette, S., (1985) . An Illustrated Guide to the Flowers of Saudi Arabia, M.E. P.A./Scorpion, London.

Gilani and Atta-ur-Rhaman, (2005) . Trends in ethno pharmacology. J. Ethnopharmacol., 100:43-49.

Mandaville, J., (1990) . The Flora of Eastern Saudi Arabia. Kegan Paul, London \& N.C. W.C. D., Riyadh.

Miller AG, Nyberg JA. (1991). Patterns of endemism in Arabia. Flora et Vegetatio Mundi, 9 263-279 
Rowinsky, E. K., N. Onetto, R. M. Canetta and R. M. Arbuck. (1992) . Taxolthe 1st of the texanes, an important new class of anti-tumor agents. Semin Oncol. 19: 646-62.

Sangwan F., (2011) . Mucilages and their Pharmaceutical Applications: an Overview. Pharmacology Online 2: 1265-1271.

Schultz, E. \& Whitney, J. (1986) . Vegetation in north-central Saudi Arabia, Jour. Arid Environ. 10: 175-288.

Shaltout, K. (1997) . Current situation of flora and vegetation of south-west Egypt. In: Integrated Land Development of Southern Egypt: Available Resources and Alternative Options. Eds. Abdel-Ghaffar, A.S., S.M. Marei and H.M. Gaber. ICDR, Canada.

Shoeb M. Anticancer agents from medicinal plants. Bangladesh J Pharmacol. 2006. 1: 35-41 Siddiqui S. and Siddiqui, R.H. (1931) . Chemical examination of root of Rouwoifia serpentina. Jour.md. Chem Soc.; 8: 667.

Tackhlom, V., (1974) . Student's Flora of Egypt. Cairo University Press, Cairo, Egypt.

Yassin, M., Salih, A., \& Hosny, A., (2013) . Medicinal Plants in Saudi Arabia: I. Sarrwat Mountains at Taif, KSA, Academic Journal of Plant Sciences 6(4): 134-145.

Yuan, X.; Chapman, R.L.; Wu, Z. (2011) . Analytical methods for heavy metals in herbal medicines. Phytochem. Anal. 22, 189-198.

http://www.citiestips.com/city/AlBahaSaudiArabia. 
دراسة مقارنة القلورة العربية السعودية والنباتات الطبية بمحافظة المندق منطقة الباحة

$$
\begin{aligned}
& \text { محمد السيد عبد الهادي * ',"، وأحمد جاديم محمد علي ؟,؛ } \\
& \text { ' قسم الأحياء ، كلية العلوم و الآداب ، المندق ، جامعة الباحة . } \\
& \text { ץ قسم الكيمياء ، كلية العلوم والآداب ، المندق ، جامعة الباحة . } \\
& \text { × قسم النبات ، كلية العلوم ، القاهرة ، جامعة الأزهر ، مصر. } \\
& \text { ؛ قسم الكيمياء ـ كلية التربية ـ جامعة السلام ـ السودان. }
\end{aligned}
$$

|البريد الاكتروني للباحث الرئيسي: yahoo.com:

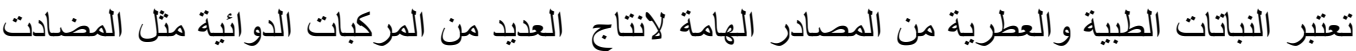

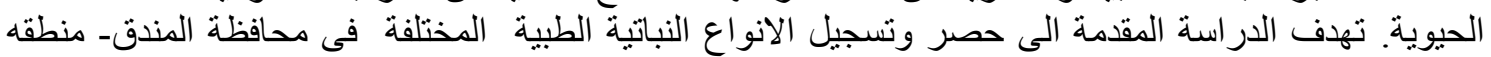

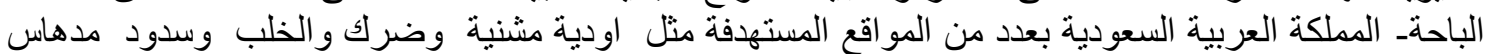

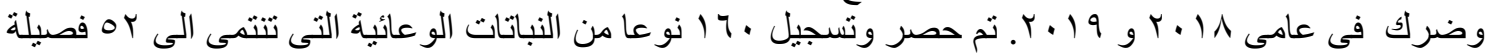

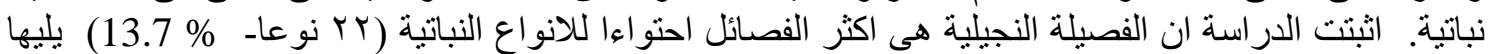

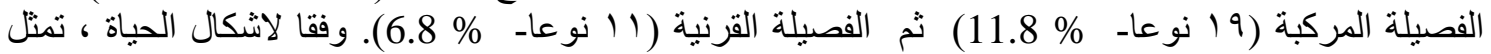

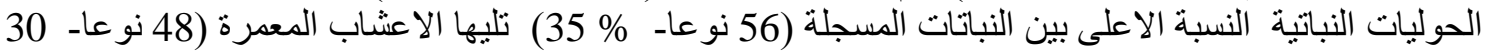

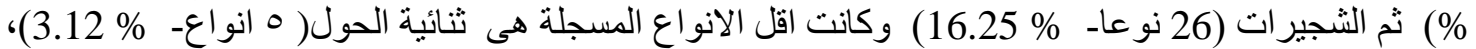

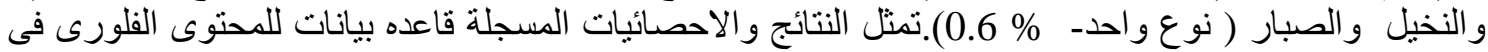

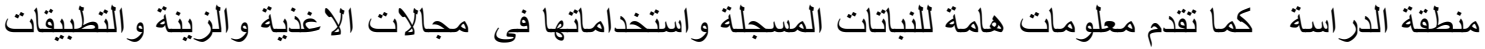
البيولوجي.

الكلمات المفتاحية: نباتات طبية ، فلورا ، فلور ا حضري ، المملكة العربية السعودية ، استخدامات اقتصادية ، 